\title{
15th European Conference on the
}

\section{Spectroscopy of Biological Molecules (ECSBM) - where spectroscopy and biology met}

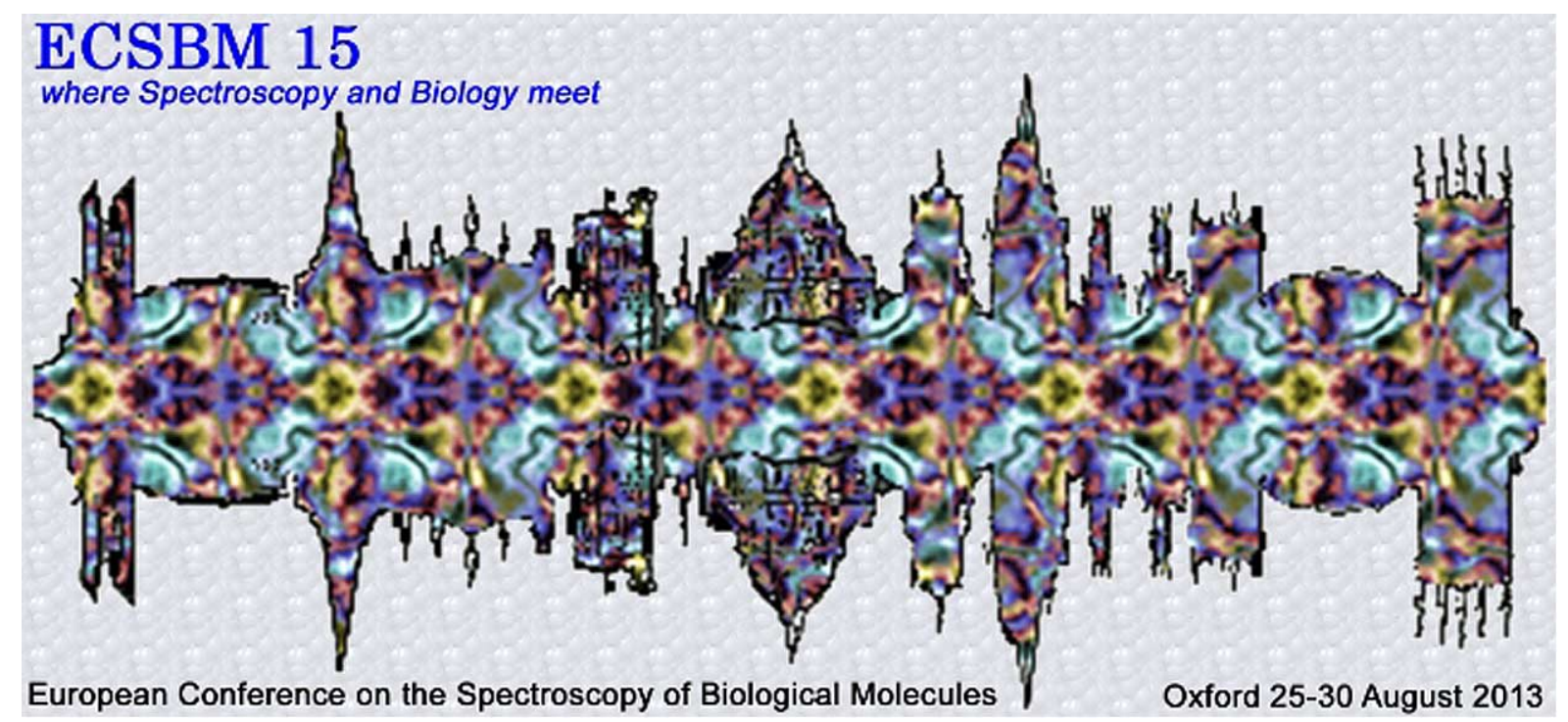

The 15th European Conference on the Spectroscopy of Biological Molecules (ECSBM) was held from 25-30 August 2013, at St Catherine's College, Oxford, England. The venue provided a stimulating background for the conference by enabling delegates to sample the traditional values of Oxford College life within contemporary architecture. The college motto, Nova et Vetera (the new and the old), reflects this perfectly and gave ECSBM 15 a distinctive location to discuss and pursue the future of our scientific heritage.

The meeting brought together a multidisciplinary community developing and using a wide range of spectroscopic techniques (Raman, IR, UV-Vis, fluorescence, CD, NMR, EPR), as well as those who work on imaging techniques applicable to the study of biomolecules, cells, or tissues and for the diagnosis of diseases. The major themes included: Spectroscopy in Imaging; Super Resolution of cells and organisms; multidimensional Spectroscopy; Time Resolved Spectroscopy \& Imaging; Theory and Systems Modelling; Bio-Medical, Bio-Chemical Application Spectroscopy; Surface Enhanced Methods \& Membrane; Disease Diagnosis and Pharmaceutics. 


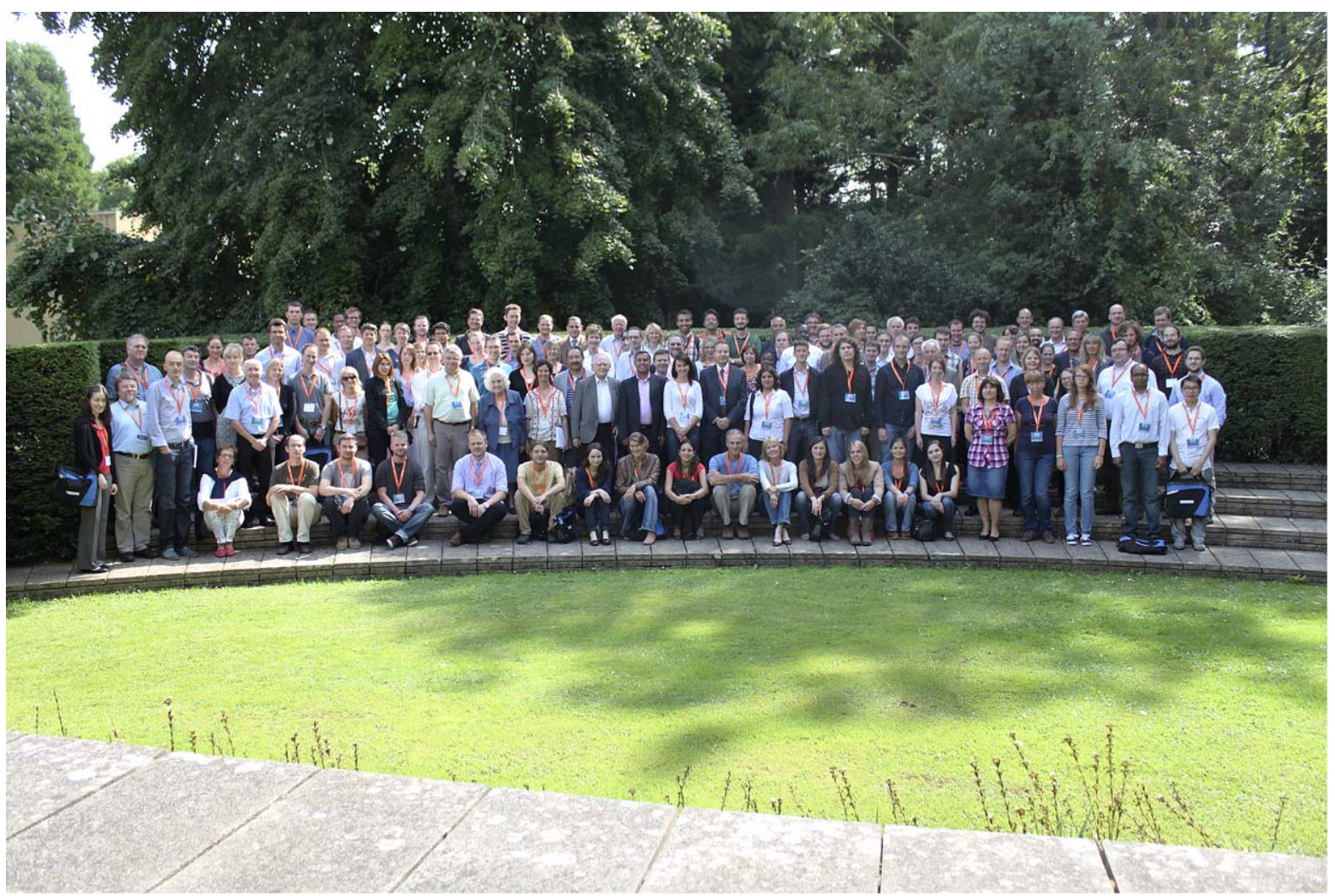

Fig. 1. Group photo of the conference participants. (Colors are visible in the online version of the article; http://dx.doi.org/ 10.3233/BSI-140090.)

Participants from the continents of Asia, Africa, Europe, North and South America attended this conference (see Fig. 1). Plenary speakers at the conference included Hugh Barr (UK), Erik Goormaghtigh (Belgium), Stefan Hell (Germany), Nick van Hulst (Spain), David Klug (UK), Roger Bisby (UK), Peter Ogilby (Denmark) and Martin Zanni (USA). Major advances in the field were presented at the meeting. Martini Zanni presented the IOS Press Biomedical Spectroscopy and Imaging sponsored lecture at the conference. This special issue of Biomedical Spectroscopy and Imaging contains a selection of work presented at the conference and includes two articles from plenary speakers Martin Zanni and High Barr. Martin Zanni and his colleagues have produced an article on their research work utilising two-dimensional infrared (2D IR) spectroscopy and demonstrate how the method can be used towards developing drugs for the treatment of Alzheimer's disease. Hugh Barr and his colleagues have produced an article on the application of Raman spectroscopy as a medical diagnostic tool.

ECSBM was founded in 1985 and is held every two years in a different European country. The meetings are attended by researchers using traditional spectroscopic techniques as well as those who work on imaging techniques in the study biological systems. Recently, particular attention has been devoted to the applications of biomolecular spectroscopy in the fields of biomedical imaging, anticancer research, drug characterization for pharmaceutical applications, drug delivery and nano-biotechnology. For its qualified attendance and for the broadness of scientific topics covered, ECSBM has become during its time a leading conference located at the crossroad of three fundamental scientific fields namely physics, 
chemistry and biology. The 16th ECSBM will be held in Bochum, Germany in September 2015 and will be chaired by Klaus Gerwert.

Anthony W. Parker Chair of the 15th ECSBM Central Laser Facility STFC Rutherford Appleton Laboratory Harwell Oxford

Didcot, Oxfordshire OX11 OQX, Oxford, UK

Parvez I. Haris Faculty of Health \& Life Sciences De Montfort University Leicester, LE1 9BH, UK 